Proceedings of the $2^{\text {nd }}$ ICEENG conference, 23-25 Nov. 1999

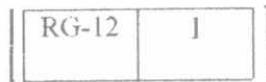

MILITARY TECHNICAL COLLEGE
Kobry Elkobbah - CAIRO - EGYPT

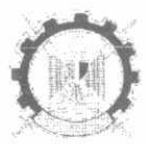

SECOND INTERNATIONAL CONFERENCE ON ELECTRICAL ENGINEERING NOVEMBER $23-25$ 1999

\title{
THREE AXIS STABILIZATION SATELLITE MODELING AND ITS CONTROL SYSTEM
}

\author{
* Gamal M.I. Selim ** Ayman Elshabrawy
}

\begin{abstract}
Satellite control is considered as one of the most modern spacecraft technology. The main advantage of satellite is its ability to obtain a global look at large area of the earth's surface, therefor a system of three satellites in geosynchronous orbit can cover almost all of the earth's surface. This led to the application of satellites in several areas, such as communications by high frequency line-of -sight radio links, photography for meteorology, earth resources and navigation for aircraft and ships. This paper introduce the design and the computer simulate of the attitude control system for three axis stabilization satellite
\end{abstract}

\section{Keyword}

Satellite, Three axes stabilization.

\section{INTRODUCTION}

The satellite consists of two main part :-

\section{The mission payload}

The mission payload performs the mission of the spacecraft such as: communication, antennas, transponder, very high resolution radiometer, earth resources scanner, ......etc.

The main payload change from satellite to another depending on the required mission. For earth resource satellite; the primary payload is a multispectral scanner (MSS) which creates simultaneous image in four spectral bands; two in the visible frequency band (green and red) and the others in the infrared band.

\section{The spacecraft bus}

Spacecraft bus can be divided into six subsystems

1. The attitude control subsystem:

which maintains the spacecraft attitude or orientation in space within the limits allowed, it consists of sensors for attitude determination and actuators such as thrusters and/or angular momentum storage devices to provide correction torque to overcome the disturbance torque's.

*Ph.D, Dept of radar and guidance ,Egyption armed force

** Msc, Egyption armed force. 
1. The propulsion subsystem

2. The electric power subsystem

3. The thermal control subsystem

4. The structural subsystem

5. The telemetry and command subsystem

This paper is organized in two sections. Section 1 discuss the different types of attitude control of satellite and its theory.

In section 3 the attitude control system for three axes stabilization satellite and its main components with the design for each component is described.

\section{2- ATTITUDE STABILIZATION METHODS}

The attitude control system use three methods for stabilization which are, spin control, dual spin control and three axes active control.

\section{2-1 Spin stabilization:}

Spin stabilization provides simplicity, low cost, high reliability, and long system life. The entire spacecraft is spun permitting fixed internal orientation. Gyroscopic resistance provide stabilization about transverse axes and thus resistance to external disturbance torque.

The disadvantages of this method are :-

1. Sensor imaging is limited to line scanning obtained by spin motion. Only one axis is oriented to a fixed reference which has poor maneuverability due to high angular momentum of the spacecraft .

2. Ground assistance for momentum precession control is normally required.

3. Power efficiency is inherently poor due to solar array being located on the spinning body since half of the array is illuminated.

\section{2-2 Dual - Spin Control:}

As in the case of a pure spinner the major portion of the dual spin controlled spacecraft is spun while only the payload section is despond. Autonomous platform pointing about the spin axis provides for continuous earth viewing from the platform.

Also dual-spin control needs ground assistance for precision control. The main advantage of this system is that fixed internal orientation with scanning and pointing capability is possible.

The disadvantage is the limited growth potential due to the inefficient solar array on the spinner, also it is more costly and complex than the first control scheme.

\section{2-3 Three Axis Active Control:}

In the three axis active spacecraft control systems the major part is despond the payload is mounted on the main body which is controlled in attitude by either a mass explosion subsystem (jets) or reaction wheels. The primary advantage of the system is that high pointing accuracy which is limited only by that which the sensing subsystem can achieve. 
Proceedings of the $2^{\text {nd }}$ ICEENG conference, 23-25 Nov. 1999

\begin{tabular}{|l|l|}
\hline RG-12 & 3 \\
\hline
\end{tabular}

Three axis control system are adaptable to changing mission, maneuvering and precision orientation requirements.

Of course the disadvantages are its high cost hardware, greater weight and power requirements.

\section{3- ATTITUDE DYNAMICS AND CONTROL}

Communication satellites require high pointing accuracy for the antennas so that it may provide the desired coverage on the earth's surface.

This requirement is achieved through an attitude control system which maintains the spacecraft attitude (its orientation in space) within the allowable limits.

A block diagram of an attitude control system is shown in Fig.1 The basic elements of an attitude control system are : -

1- Spacecraft attitude dynamics.

2- Attitude sensor.

3- Control law.

4- Actuator.

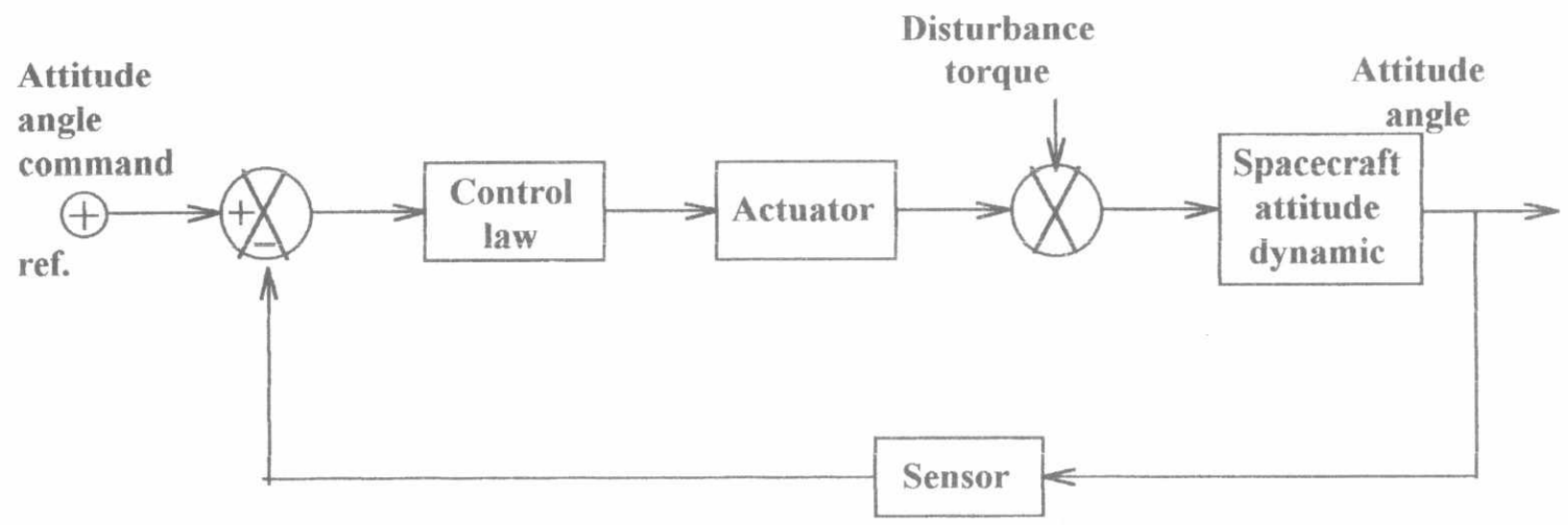

Fig. 1 Block diagram of an attitude control system

Orbit dynamics deals with the motion of the center of mass of a spacecraft, while attitude dynamics is concerned with the rotational motion of the spacecraft body about its center of mass.

In this study it is assumed that the spacecraft is rigid (which is not really true) of course, the equations of motion derived for rigid spacecraft will be modified for flexible spacecraft. 


\section{3-1 Three Axis Stabilization:}

There are two main types of three axis stabilization systems ":

1- momentum based system with a momentum wheel along the systems.

2- Zero momentum system with reaction wheel along each axis.

Define the three axis coordinate system as roll, pitch, and yaw.

The nominal " roll axis $X$ " is along the orbit velocity.

The nominal "yaw axis $Z$ " is along the vector from the center of mass of spacecraft to center of mass of the earth.

The nominal pitch axis $Y$ is normal to the orbit plane.

In case of momentum wheel system, the angular momentum along the pitch axis provides gyroscopic stiffness.

Pitch and roll axes are controlled directly, while the yaw axis is controlled indirectly thus eliminating the need for yaw sensor.

\section{3-2 The External Momentum}

The external momentum's arises from three major sources .

1-The gravitational gradient

2- The solar radiation pressure

3- The control momentum from actuator

$$
M=M_{G}+M_{s}+M_{C}
$$

$\mathrm{M}_{\mathrm{G}}$

$\mathrm{M}_{\mathrm{S}}$

$\mathrm{M}_{\mathrm{C}}$

The gravity gradient moment according to reference [1][2] is given by :

$\mathbf{M}_{\mathrm{G}}=\mathbf{3} \omega_{\mathrm{o}}{ }^{2}\left[\begin{array}{l}\phi\left(I_{z z}-I_{y y}\right) \\ \theta\left(I_{z z}-I_{x x}\right) \\ 0\end{array}\right]$

solar pressure moment $M_{S}$ into coordinate system $X_{0}, Y_{0}, Z_{0}$ which rotates $1 \%$ day is given by [1] [2]

$$
M_{s}=P A\left[\begin{array}{l}
\left(Y K_{1}-Z K_{2} \cos \alpha-X K_{2} \sin \alpha\right) I_{o} \\
\left(Z K_{1} \sin \alpha-X K_{1} \cos \alpha\right) J_{o} \\
\left(-Z K_{2} \sin \alpha+X K_{2} \cos \alpha\right) K_{o}
\end{array}\right]
$$

Where $\alpha \ldots$ is the orbital angle measured from spacecraft local noon.

\section{3-3 Fixed Momentum Wheel with Thrusters:-}

Using fixed momentum wheel with three axis stabilization neglect the need for an error command of attitude correction. The thruster is fired by on board control electronics automatically. The momentum wheel is nominally aligned with the pitch axis with the angular momentum vector along the negative pitch axis.

So, the angular momentum of the wheel is given by 
Proceedings of the $2^{\text {nd }}$ ICEENG conference, 23-25 Nov. 1999

$$
\begin{aligned}
& h_{x}=0 \quad h_{y}=-h \quad h_{z}=0 \\
& \text { where ... h }=I_{\omega} \Omega \\
& l_{\omega} \ldots . \text { is the moment of inertia. } \\
& \Omega \ldots . \text { is the angular velocity. }
\end{aligned}
$$

The momentum equation is given by [1][2][3]

$$
\left[\begin{array}{l}
M_{c x}+M_{s x} \\
M_{c y}+M_{s y} \\
M_{c z}+M_{s z}
\end{array}\right]=\left[\begin{array}{l}
I_{x x} \ddot{\phi}+\left[4 \omega_{o}^{2}\left(I_{y y}-I_{z}\right)+\omega_{o} h\right] \phi+\left[h-\omega_{o}\left(I_{x x}-I_{y y}+I_{z z}\right)\right] \dot{\psi} \\
I_{y y} \ddot{\theta}+3 \omega_{o}^{2} \theta\left(I_{x x}-I_{z z}\right)-\hat{h} \\
I_{z z} \ddot{\psi}+\left[\omega_{o}^{2}\left(I_{y y}-I_{x x}\right)+\omega_{o} h\right] \psi-\left[h-\omega_{o}\left(I_{x x}+I_{z}-I_{y y}\right)\right] \dot{\phi}
\end{array}\right]
$$

If $\mathrm{h}$ is large the moment equation is

$$
\left[\begin{array}{l}
M_{c x}+M_{s x} \\
M_{c y}+M_{s y} \\
M_{c z}+M_{s z}
\end{array}\right]=\left[\begin{array}{l}
I_{x x} \ddot{\phi}+\omega_{o} h \phi+h \psi \\
I_{y y} \ddot{\theta}+3 \omega_{o}^{2} \theta\left(I_{x x}-I_{z z}\right)-\dot{h} \\
I_{z z} \ddot{\psi}+\omega_{o} h \psi-h \dot{\phi}
\end{array}\right]
$$

Which is the general equation of motion of three axis stabilization satellite from this we conclude that pitch axis is uncoupled from the equation of the roll and yaw axis.

\section{3-4 Pitch Axis Control :}

From the momentum equation of motion derived, it is clear that :

$$
M_{c y}+M_{s y}=I_{y y} \ddot{\theta}+3 w_{o}^{2}\left(I_{x x}-I_{z z}\right) \theta-h
$$

In case of symmetric satellite $\quad I_{x x}=I_{z z}$ thus,

$$
M_{c y}+M_{s y}=I_{\psi \psi} \theta^{*}-h^{*}
$$

The pitch error $\theta$ can be controlled by applying a torque proportional to the pitch error and its rate $\dot{\theta}$. Therefore changing the angular momentum of the wheel $\dot{h}$ this can be expressed by :

$$
\dot{h}=-k_{\theta}\left(\tau_{\theta} \theta^{\bullet}+\theta\right)
$$

As $\quad \mathrm{M}_{\mathrm{sy}}=0 \quad$ the pitch equation is given by

$$
M_{s y}=I_{y y} \ddot{\theta}+k_{\theta} \tau_{\theta} \dot{\theta}+k_{\theta} \theta
$$

This is the form of famous second order system given by : 
Proceedings of the $2^{\text {nd }}$ ICEENG conference, 23-25 Nov. 1999

\begin{tabular}{|l|l|}
\hline $\mathrm{RG}-12$ & 6 \\
\hline
\end{tabular}

$$
\begin{aligned}
& s^{2}+2 \xi \omega_{n} s+\omega_{n}^{2}=0 \\
& \text { Thus } \quad \omega_{\theta}=\sqrt{\frac{k_{\theta}}{I_{y y}}} \quad \xi_{\theta}=\frac{\tau_{\theta}}{2} \sqrt{\frac{k_{\theta}}{I_{y y}}}
\end{aligned}
$$

The block diagram of the pitch axis control is shown in Fig.2

Note that momentum wheel is intended to apply a moment to the spacecraft to nullify the external moment

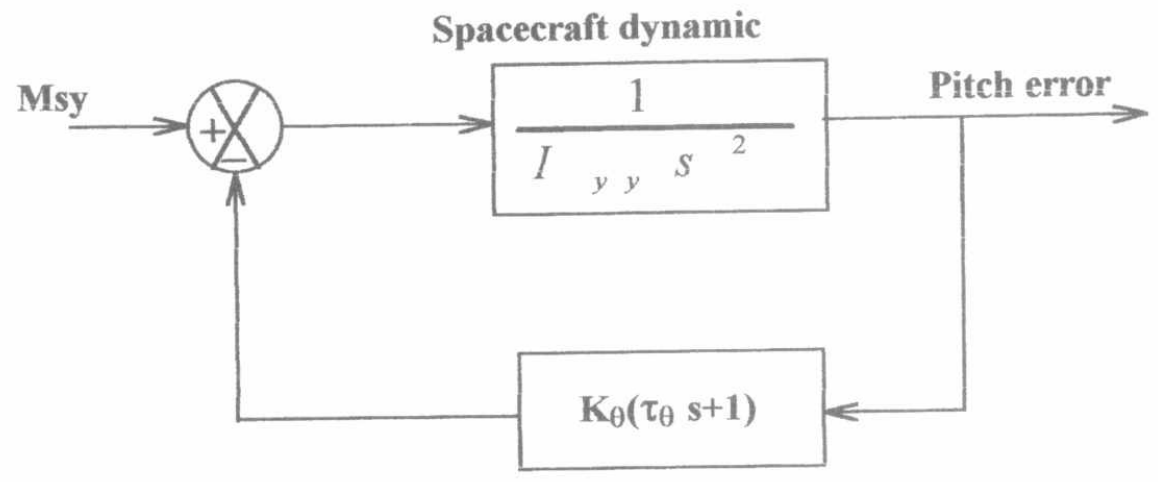

Wheel torque

Fig. 2 Block diagram for pitch axis control

The forward and the feedback transfer function are given by

$$
\begin{aligned}
& \mathrm{G}(\mathrm{s})=\frac{1}{I_{y y} s^{2}} \\
& H(s)=k_{\theta}\left(\tau_{\theta} s+1\right)
\end{aligned}
$$

Therefore the open - loop transfer function $\quad \mathrm{G}(\mathrm{s}) \mathrm{H}(\mathrm{s})$ can be written as

$$
G(s) H(s)=\frac{k_{\theta}\left(\tau_{\theta} s+1\right)}{I_{y y} s^{2}}
$$

The root locus plot of this system is shown in Fig. 3

The design point is selected to be the intersection of root locus with real axis (break in point), since the system is critically damped at this point $\left(\xi_{\theta}=1\right)$.

$$
\tau_{\theta}=2 \sqrt{\frac{I_{y y}}{K_{\theta}}}
$$


Proceedings of the $2^{\text {nd }}$ ICEENG conference, 23-25 Nov. 1999
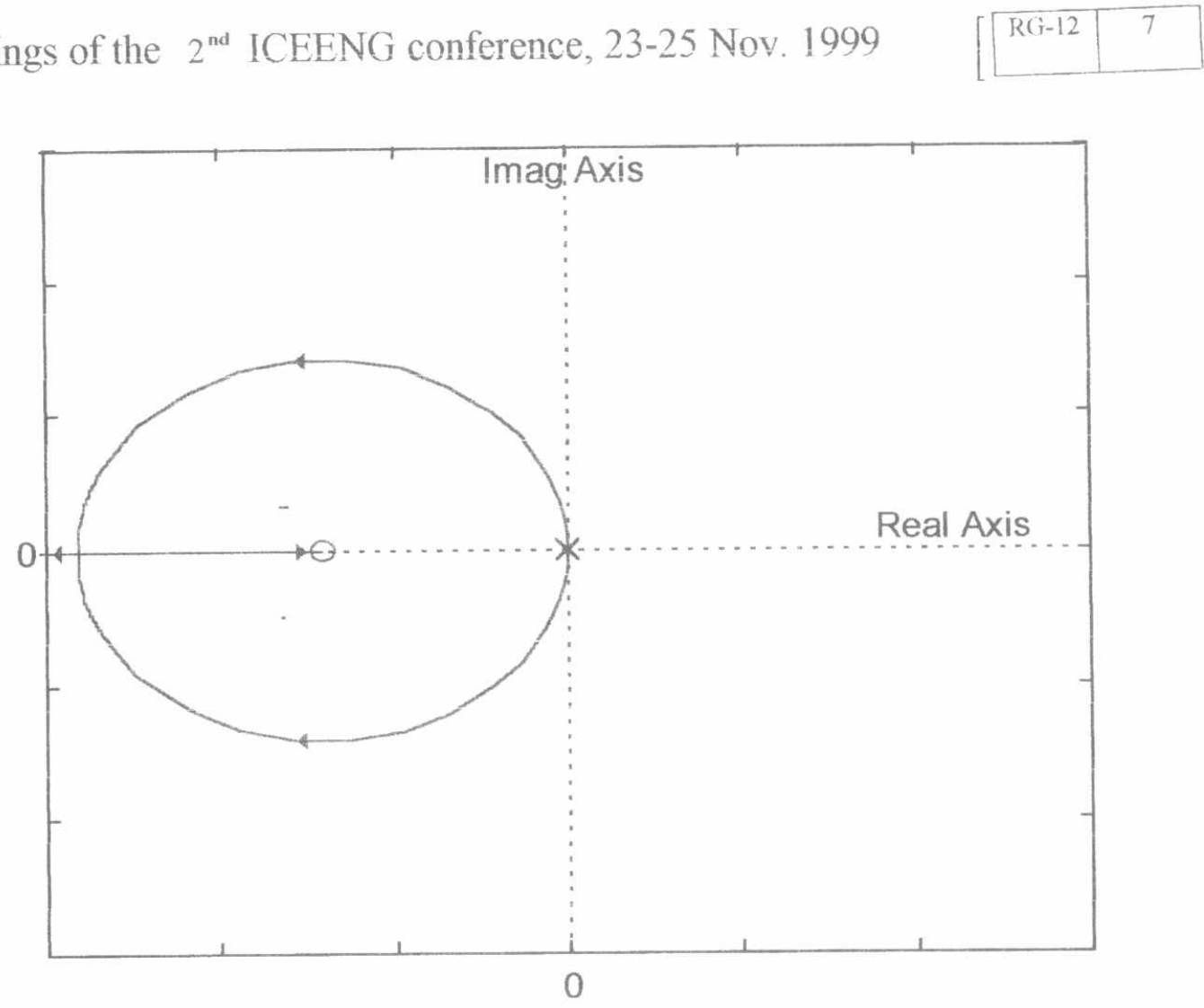

Fig.3 Root locus plot for pitch axis control

The closed loop transfer function will be

$$
\begin{aligned}
\frac{\theta(s)}{M_{s y}(s)} & =\frac{1}{I_{y y} s^{2}+k_{\theta} \tau_{\theta} s+k_{\theta}} \\
& =\frac{1}{\mathrm{I}_{\mathrm{y} y}\left(\mathrm{~s}+\sqrt{\frac{\mathrm{k}_{\theta}}{\mathrm{I}_{\mathrm{y} y}}}\right)^{2}}
\end{aligned}
$$

The selection of pitch autopilot gain $\mathbf{k}_{\theta}$ is based on two considerations ; steady state error and response time.

The pitch error is introduced from several sources, such as initial pitch error, impulse moments from a thruster during desaturation period (to keep the wheel speed within allowable range )and during station keeping because of the misalignment of the thrusters, and cyclic moment due to solar pressure

The parameters of the control system, such as $K_{\theta}$ and $\tau_{\theta}$ are selected such that the attitude error due to these disturbances are within aliowable limits

\section{3-4 The Roll -Yaw Loop Control :}

Taking the laplace transform of equation of motion for roll_yaw channel gives 
Proceedings of the $2^{\text {nd }}$ ICEENG conference, 23-25 Nov. 1999

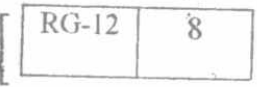

$$
\left[\begin{array}{l}
M_{c x}+M_{s y} \\
M_{c z}+M_{s z}
\end{array}\right]=\left[\begin{array}{ll}
I_{x x} s^{2}+\omega_{o} h & h s \\
-h s & I_{Z Z} s^{2}+\omega_{o} h
\end{array}\right]\left[\begin{array}{l}
\phi(s) \\
\psi(s)
\end{array}\right]
$$

For uncontrolled spacecraft

$$
M_{\mathrm{cx}}=M_{\mathrm{cz}}=0
$$

Where

$\omega_{\text {o }} \ldots . . .$. orbital rate

The transfer function between yaw angle and the yaw torque is given by :

$$
\frac{\psi(s)}{M_{z}(s)}=\frac{I_{x x} s^{2}+\omega_{o} h}{\left(I_{x x} s^{2}+\omega_{o} h\right)\left(I_{x x} s^{2}+\omega_{o} h\right)+h^{2} s^{2}}
$$

The characteristic equation can be written as :

$$
s^{4}+\left(2 \omega_{o} \omega_{n}+\omega_{n}^{2}\right) s^{2}+\omega_{o}^{2} \omega_{n}^{2}=o
$$

where:

$$
\omega_{n}=h / I_{x x} \ldots \ldots . \text { is the nutation frequency. }
$$

After every quarter period of orbital period the yaw and roll errors interchange. Therefor it is necessary to sense and control one error directly either yaw or roll and the other error is controlled indirectly due to the coupling effect.

It is well known for satellite designers that the roll error has a greater impact on the antenna pointing accuracy and it is easier to sense than the yaw error. The roll error is sensed and controlled directly and the yaw error is controlled indirectly.

Several approaches are established for roll-yaw control [1],[2]. In this paper it is decided to use the WHECON control system approach [1]. In this method the control torque is applied along the roll axis by control jet and it is proportional to the roll error $\phi$ and the roll error rate $\phi$.

The control jets are commanded only on or off so a modulator is necessary to transform the control signal to the on - off signal for control jets. The common practice is to use a pseudorate modulator. A schmitt trigger with hysteresis and real pole feedback produces a train of pulses whose average is proportional to the error and the error rate.

The control torque along the roll and yaw axes are given by :

$$
\begin{aligned}
& M_{c x}=-k \cos \alpha(\tau s+1) \phi(s) \\
& M_{c z}=k \sin \alpha(\tau s+1) \phi(s)
\end{aligned}
$$

where

k Is the gain determined by thrust force

$\alpha \quad$ Is the effect of angle of thruster

$\tau$ Is the lead time constant of pseudorate circuit

The equation of motion in the roll-yaw axis is : 


$$
\left.\left[\begin{array}{c}
M_{s y} \\
M_{s z}
\end{array}\right]=\left[\begin{array}{rr}
-I_{x x} s^{2}+\omega_{o} h+K \cos \alpha(\tau s+1) & h s \\
-k \sin \alpha(\tau s+1) & I_{z z} s^{2}+\omega_{o} h
\end{array}\right] \begin{array}{l}
\phi(s) \\
\psi(s)
\end{array}\right]
$$

$\mathrm{C}$

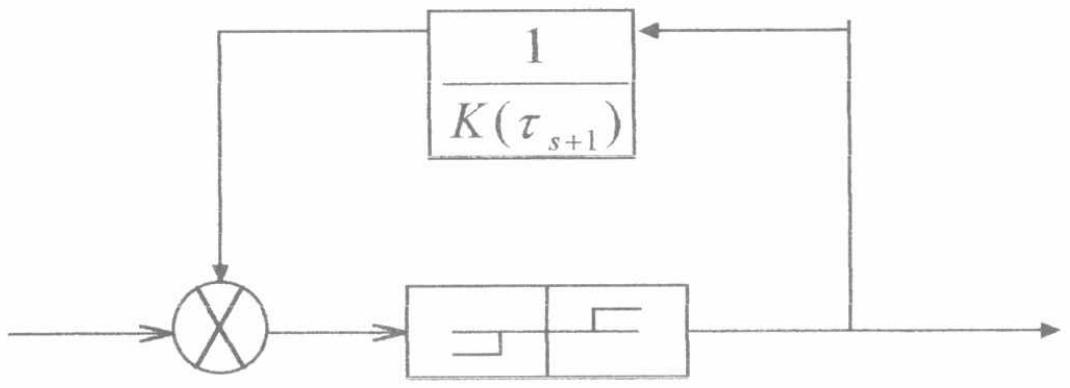

attitude error $\left(\tau_{\mathrm{s}+1}\right) \varepsilon$

\section{Schmitt trigger with}

hysteresis
$\mathrm{M}_{\mathrm{C}}=\mathrm{K}$

Fig. 4 Block diagram of pseudorate modulation

The corresponding characteristic equation is given by :

$s^{4}\left(I_{x \alpha} I_{z z}\right)+s^{3}\left(k \tau I_{z z} \cos \alpha\right)+s^{2}\left[h\left(\omega_{0} I_{x x}+\omega_{0} I_{z z}+h+k \tau \sin \alpha\right)+k \cos \alpha I_{z z}\right]$

$+s\left[h\left(k \tau \omega_{0} \cos \alpha\right)\right]+\left(\omega_{0}^{2} h^{2}+\omega_{0} h k \cos \alpha\right)=0$

According to Routh criterion a necessary-but not sufficient- condition for stability is that all coefficients of characteristic equation be positive.

Therefor $\quad k, \quad \tau, \quad \alpha, \quad$ and $h$ are all positive. The angular momentum of the wheel is determined mainly by the required yaw accuracy .

In the absence of disturbance and control torque the orbital roll-yaw cycle is a circle.

The open loop transfer function of the roll axis is given by

$$
G(s) H(s)=\frac{k I_{z z} \cos \alpha\left(s+\omega_{O} / \tan \alpha\right)\left(s+h \tan \alpha / I_{z z}\right)\left(\tau_{s}+1\right)}{I_{z z} I_{x x}\left(s^{2}+h^{2} / I_{x x} I_{z z}\right)\left(s^{2}+\omega_{o}^{2}\right)}
$$

The root locus of the closed loop roll - yaw control is shown in Fig. 5

$$
\begin{array}{lr}
\omega_{1}=\sqrt{\frac{k \cos \alpha}{N I_{x x}}} & \xi_{1}=\frac{\tau}{2} \sqrt{\frac{N k \cos \alpha}{I_{x x}}} \\
\omega_{2}=\sqrt{\frac{N \omega_{o} h}{I_{z z}}} & \xi_{2}=\frac{\tan \alpha}{2} \sqrt{\frac{N h}{I_{z z} \omega_{o}}}
\end{array}
$$

Which are the closed loop natural frequencies and associated damping ratio.

Where $N=\frac{1}{1+h^{2} / I_{z z} k \cos \alpha}$ 
Proceedings of the $2^{\text {nd }}$ ICEENG conference, 23-25 Nov. 1999

So to provide high damping and rapid response to disturbance input the values of $\tau$ , are nominally chosen so that the system is critically damped. The thruster offset angle $\alpha$ is selected to critically damp the orbital frequency mode $\omega_{2}$. Setting $\xi_{2}$ $=1$ will lead to the design equation of $\alpha$

$$
\alpha=\tan ^{-1} 2 \sqrt{\frac{I_{z z} \omega_{o}}{N h}}
$$

The time lead constant, $\tau$, is selected to critically damped notation frequency motion setting $\xi_{1}=1$ will give the design equation for $\tau$

$$
\tau=2 \sqrt{\frac{I_{x x}}{N k \cos \alpha}}
$$

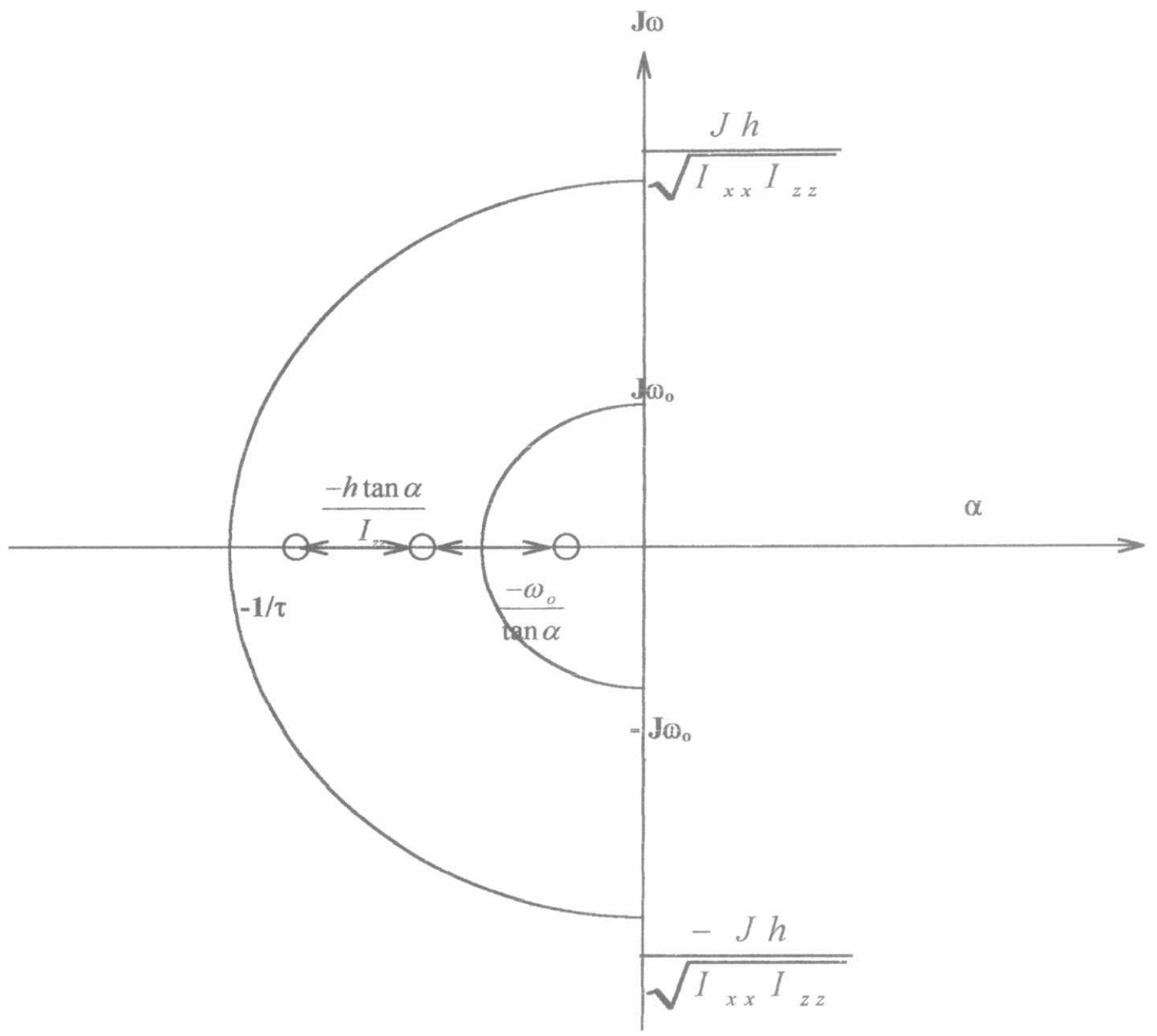

Fig.5 Root locus for roll-yaw coupling 
Proceedings of the $2^{\text {md }}$ ICEENG conference, 23-25 Nov. 1999

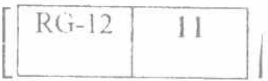

\section{Model Specifications}

For three axis stabilization spacecraft with a fixed momentum wheel with thrusters whose properties are:

$$
\begin{array}{ll}
I_{x x}=2700 \quad \mathrm{Kg} \mathrm{m}^{2} & \\
I_{y y}=1360 \quad \mathrm{Kg} \mathrm{m}^{2} & \\
\mathrm{I}_{z z}=2200 \quad \mathrm{Kg} \mathrm{m}^{2} & \\
A=16.3 \mathrm{~m}^{2} & \text { solar array area. } \\
\rho_{\mathrm{s}}=0.2 & \text { coefficient of specular reflection. } \\
\rho_{\mathrm{d}}=0 & \text { The coefficient of diffuse reflection. }
\end{array}
$$$$
\rho_{\mathrm{s}}=0.2 \quad \text { coefficient of specular reflection. }
$$

The components of $\mathrm{CM}-\mathrm{CP}$ offset are

$$
\begin{array}{ll}
x=0.03 \quad m \\
y=0.03 & m \\
z=-0.33 & m
\end{array}
$$

Roll thruster torque $=0.615 \mathrm{~N} \mathrm{~m}$.

Wheel desaturation thrust torque $=0.422 \mathrm{~N} \mathrm{~m}$. with pulse time of $0.2 \mathrm{sec}$.

The permissible attitude control errors are $0.04^{\circ}$ in roll and pitch and $0.04^{\circ}$ in yaw.

Linear range of sensor $= \pm 3^{\circ}$

As $\delta$ is the declination of the sun so at equinox $\delta=0$

$$
\begin{aligned}
& k_{1}=\left[\left(1-\rho_{s}\right) \cos \delta+2\left(\rho_{s}+\frac{1}{3} \rho_{d}\right)\right] \cos \delta \\
& k_{2}=\left(1-\rho_{s}\right) \cos \delta \sin \delta \\
& \mathrm{k}_{1}=1+\rho_{\mathrm{s}}=1.2 \\
& \mathrm{k}_{2}=0
\end{aligned}
$$

The solar torque is determined as

$$
\begin{aligned}
& \mathrm{M}_{\mathrm{s}}=\operatorname{PA}\left[\begin{array}{c}
\left(\mathrm{yk}_{1} \cos \alpha-\mathrm{zk}_{2}\right) \mathrm{I} \\
\left(\mathrm{zk}_{1} \sin \alpha-\mathrm{xk}_{1} \cos \alpha\right) \mathrm{J} \\
\left(\mathrm{xk}_{2}-\mathrm{yk}_{1} \sin \alpha\right) \mathrm{k}
\end{array}\right] \\
& \mathrm{M}_{\mathrm{s}}=4.644 * 10^{-6} * 16.3\left[\begin{array}{l}
(0.03 * 1.2 \cos \alpha) \mathrm{I} \\
(-0.33 * 1.2 \sin \alpha-0.03 * 1.2 \cos \alpha) \mathrm{J} \\
(-0.03 * 1.2 \sin \alpha) \mathrm{K}
\end{array}\right] \\
& \mathrm{M}_{\mathrm{s}}=\left(2.72 * 10^{-6} \cos \alpha\right) \mathrm{I}+\left(3 * 10^{-5} \sin \alpha-2.72 * 10^{-6} \cos \alpha\right) \mathrm{J} \\
& -\left(2.72 * 10^{-6} \sin \alpha\right) \mathrm{K}
\end{aligned}
$$


Proceedings of the $2^{\text {nd }}$ ICEENG conference, 23-25 Nov. 1999

At summer solstice the same equation as before, except for $\delta=23.5^{\circ}$

$$
\begin{aligned}
& \mathrm{k}_{1}=[(1-0.2) \cos 23.5+2(0.2+0)] \cos 23.5=1.039 \\
& \mathrm{k}_{2}=(1-0.2) \cos 23.5 \sin 23.5=0.293 \\
& \mathrm{M}_{\mathrm{s}}=4.644 * 10^{-6} * 16.3\left[\begin{array}{l}
(0.03 * 1.039 \cos \alpha+0.33 * 0.293) \mathrm{I} \\
(-0.33 * 1.039 \sin \alpha-0.03 * 1.039 \cos \alpha) \mathrm{J} \\
(0.03 * 0.293-0.03 * 1.039 \sin \alpha) \mathrm{K}
\end{array}\right]
\end{aligned}
$$

So,

$$
\begin{aligned}
M_{s}= & \left(2.36 * 10^{-6} \cos \alpha+7.3 * 10^{-6}\right) I-\left(2.6^{*} 10^{-5} \sin \alpha+2.36 * 10^{-6} \cos \alpha\right) J \\
& +\left(0.664 * 10^{-6}-2.36 * 10^{-6} \sin \alpha\right) K
\end{aligned}
$$

In initial coordinates the solar torque are determined by equation

$$
\mathrm{M}_{\mathrm{s}}=\operatorname{PA}\left[\begin{array}{l}
\left(\mathrm{Yk}_{1}-\mathrm{Zk}_{2} \cos \alpha-\mathrm{Xk}_{2} \sin \alpha\right) \mathrm{I}_{\mathrm{o}} \\
\left(\mathrm{Zk}_{1} \sin \alpha-\mathrm{Xk}_{1} \cos \alpha\right) \mathrm{J}_{\mathrm{o}} \\
\left(-\mathrm{Zk}_{2} \sin \alpha+\mathrm{Xk}_{2} \cos \alpha\right) \mathrm{K}_{\mathrm{o}}
\end{array}\right]
$$

So, solar torque at equinox will be

$$
\mathrm{M}_{\mathrm{s}}=\left(2.72 * 10^{-6}\right) \mathrm{I}_{\mathrm{o}}+\left(3 * 10^{-5} \sin \alpha-2.72 * 10^{-6} \cos \alpha\right) \mathrm{J}_{\mathrm{o}}
$$

And at summer solstice the equation is given by

$$
\begin{aligned}
\mathrm{M}_{\mathrm{s}}= & \left(2.36 * 10^{-6}+7.3 * 10^{-6} \cos \alpha-0.66 * 10^{-6} \sin \alpha\right) \mathrm{I}_{\mathrm{o}} \\
& -\left(2.6 * 10^{-5} \sin \alpha+2.36 * 10^{-6} \cos \alpha\right) \mathrm{J}_{\mathrm{o}} \\
& +\left(7.3 * 10^{-6} \sin \alpha+0.66 * 10^{-6} \cos \alpha\right) \mathrm{K}_{\mathrm{o}}
\end{aligned}
$$

Let the constant roll - yaw solar torque in inertial coordinate at equinox be

$$
\mathrm{T}_{\mathrm{s}}=1.77^{*} 10^{-6} \mathrm{~N}
$$

\section{The Roll-yaw Control Channel}

The gain $k$ (output gain) is the maximum control torque available divided by the linear range of sensor, So

$$
k=\frac{0.165 * 180}{3 \pi}=11.74 \quad \mathrm{Nm} / \mathrm{rad}
$$

The steady state error of yaw is given by : 


$$
\begin{aligned}
& \psi_{\mathrm{ss}}=\frac{T_{b z}}{\omega_{\mathrm{o}} h} \\
& \mathrm{~h}=\frac{1.77 \times 10^{-6} \times 180 \times 24 \times 60 \times 60}{2 \Pi \times 0.04 \times \Pi}
\end{aligned}
$$$$
=35 \text { N.M.S }
$$

Since $\alpha$ is small then $\cos \alpha \cong 1$

$$
\begin{aligned}
& \mathrm{N}=\frac{1}{1+\mathrm{h}^{2} / \mathrm{I}_{z \mathrm{z}} \mathrm{k}} \\
& \mathrm{N}=\frac{1}{1+(35)^{2} /(2200 * 11.74)}=0.955
\end{aligned}
$$

The offset angle of the thruster is

$$
\begin{aligned}
& \alpha=\tan ^{-1} 2 \sqrt{\frac{\mathrm{I}_{27} \omega_{\mathrm{o}}}{\mathrm{Nh}}} \\
& \alpha=\tan ^{-1} 2 \sqrt{\frac{2200 * 2 \pi}{24 * 60 * 60 * 0.955 * 35}}=7.8^{\circ} \\
& \omega_{o}=\frac{2 \pi}{f}=\frac{2 \pi}{24 * 60 * 60}
\end{aligned}
$$

The lead time constant $\tau$ is selected using design equation

$$
\begin{aligned}
& \tau=2 \sqrt{\frac{I_{x x}}{N k \cos \alpha}}=2 \sqrt{\frac{2700}{0.955 * 11.74 * 0.99}}=31.2 \quad \text { sec. } \\
& M_{c y}=k \cos \alpha\left(\tau_{s}+1\right) \phi(s)
\end{aligned}
$$

\section{The Pitch Control:-}

The desaturation torque impulse $\mathrm{M}_{\mathrm{y}}$ will be $M_{\mathrm{y}}=0.422 * 0.2=0.0844 \quad \mathrm{~N} \mathrm{~m} \mathrm{~s}$

The pitch control design is based on the maximum allowable pitch error. So the time constant of the system can be obtained from the equation

$$
\theta_{\text {max }}=\frac{M_{y} \tau}{I_{y y}} \frac{1}{e}
$$


Proceedings of the $2^{\text {nd }}$ ICEENG conference, 23-25 Nov. 1999

$$
\begin{aligned}
\tau & =\frac{\theta_{\max } \mathrm{I}_{\mathrm{yy}} \mathrm{e}}{\mathrm{M}_{\mathrm{y}}}=\frac{0.04 * \pi}{180} * \frac{1360 * 2.718}{0.0844}=30.57 \\
\tau & \cong 31 \mathrm{sec} .
\end{aligned}
$$

This time is defined as

$$
\tau=\sqrt{\frac{I_{y}}{K_{\theta}}}
$$

Thus

$$
K_{\theta}=\frac{I_{y y}}{\tau^{2}}=1.41 \quad N . m / r a d
$$

Which is pitch lead gain, the lead time constant $\tau_{\theta}$ is

$$
\tau_{\theta}=2 \sqrt{\frac{I_{y y}}{K_{\theta}}}=62 \quad \text { sec }
$$

The open loop transfer function of pitch is given by

$$
G(s)=\frac{1}{1360 \mathrm{~S}^{2}}
$$

And the feed back transfer function is given by :

$H(s)=1.41(62 \mathrm{~S}+1)$

The output time response for pitch angle is shown in Fig. 6

Obtain the transfer function of roll axis with the controller.

$$
\left[\begin{array}{l}
M_{s x} \\
M_{s z}
\end{array}\right]=\left[\begin{array}{ll}
I_{x x} s^{2}+\omega_{o} h+k \cos \alpha\left(\tau_{s}+1\right) & h s \\
-k \sin \alpha\left(\tau_{s}+1\right)-h s & I_{z z} s^{2}+\omega_{o} h
\end{array}\right]\left[\begin{array}{l}
\phi(s) \\
\psi(s)
\end{array}\right]
$$

when substituting the numerical value of our model then the roll open loop transfer function is

$$
\mathrm{G}(\mathrm{s})=\frac{2200 \mathrm{~s}^{2}+2.54 \times 10^{-3}}{594 \times 10^{4} \mathrm{~s}^{2}+1237.4 \mathrm{~s}^{2}+6.4516 \times 10^{-6}}
$$

Where the feedback controller is

$H(s)=11.74 \cos 7.8(31.3 s+1)$

The time response of roll angle and controller output due to disturbance torque of $8 * 10^{-5}$ is shown in Fig. 7 
Proceedings of the $2^{\text {nd }}$ ICEENG conference, 23-25 Nov. 1999

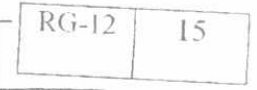

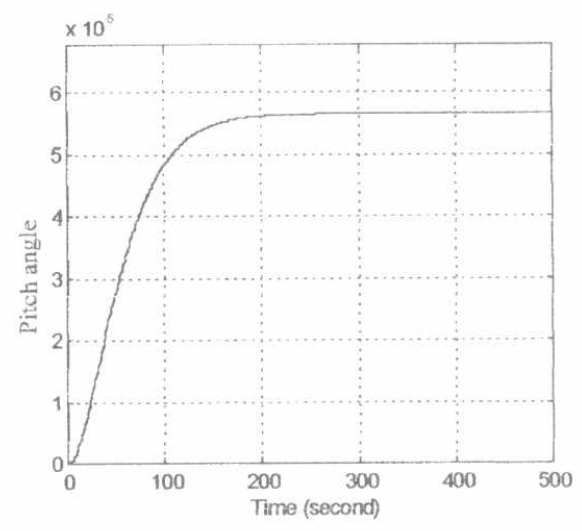

Fig. 6 The response of pitch angle

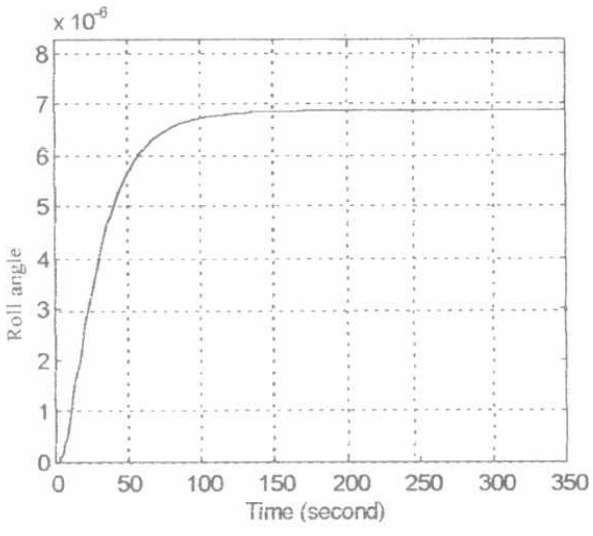

Fig.7 The response of roll angle

\section{CONCLUSION}

A Controllers for both of wheel system in pitch channel and for thruster firing in Roll/yaw channel has been designed and tested using simulation program for intelsat-v modern system satellite.

\section{REFERENCES}

[1] B.N.Agrawal " Design of geosynchronous spacecraft " Printice -hall

[2] M.H.Kaplan " Modern spacecraft dynamics and control " John wiley \& sons

[3] A.E.Sabroff " advanced spacecraft stabilization and control techniques " journal of spacecraft and rockets, Vol. [5] No 12 p.1377-1393 Dec. 1968

[4] P.P. Iwens, A.W.Fleming and V.A.Spector " precision attitude control with a single body fixed momentum wheel " AIAA Mechanics and control of flight conference, 1974, p 79-89

[5] Robert lafore " C Programming using turbo c++ " The waite group,inc.

[6] M.S. Hodgart, P.S.Wright "Attitude determination, control and stabilization of UoSAT-2. " journal of institution of electronic and radio engineers vol.57, No. ,pp151-162

[7] R.H.Canon, Jr. , "Some basic response relations for reaction wheel attitude control ", Ars journal ,jan. 1962. 